\title{
Leon Lederman (1922-2018)
}

Physicist who expanded the family tree of fundamental particles.

\section{L} eon Max Lederman's stunning discoveries, leadership and advocacy laid the foundations for particle physics today. His discovery of the muon neutrino established that there was more than one type of neutrino. His observation of muon decay knocked down a pillar theory about symmetry in one of the fundamental forces. His discovery of the long-lived neutral kaon meson helped to home in on one of the great mysteries of physics. And his discovery of bottom quarks - subatomic particles that make up neutrons and protons - led researchers to uncover a third family of quarks.

Self-effacing, approachable and imaginative, Lederman was a consummate joke-teller. "Physics is not religion," he used to quip. "If it were, we would have a much easier time raising money." He had good taste in research problems and a gift for recognizing connections and opportunities. He was also a charismatic communicator; in later years, he focused on advancing science education. $\mathrm{He}$ died on 3 October in Rexburg, Idaho, aged 96.

Lederman was born on 15 July 1922 in New York City to Jewish parents who had emigrated from Russia and who were keen on education. Lederman grew up at a time when Jewish scientists were fleeing imminent war in Europe for the United States, and he was attracted to the excitement surrounding the twentieth-century physics revolution of which many scientists were a part.

He did his bachelor's degree in chemistry at the City College of New York in 1943. After three years of US Army service in Europe, he earned his $\mathrm{PhD}$ in physics from Columbia University in New York City in 1951.

There, he spent three decades teaching and conducting experimental research as a faculty member. Nature was ripe for discovery, and clever experiments with particles accelerated to the highest energies were expected to yield the biggest discoveries.

From his mentor at Columbia, Nobelprizewinning physicist Isidor Isaac Rabi, Lederman learnt to distinguish observation (a hint of something new) from measurement (a more precise endeavour). Lederman became the master of both, with the creativity to devise unique experiments and the tenacity to follow through on them.

Lederman's most famous work was done in 1962 at Brookhaven National Laboratory in Long Island, New York, with two of his Columbia University colleagues, Jack Steinberger and Mel Schwartz. In what would

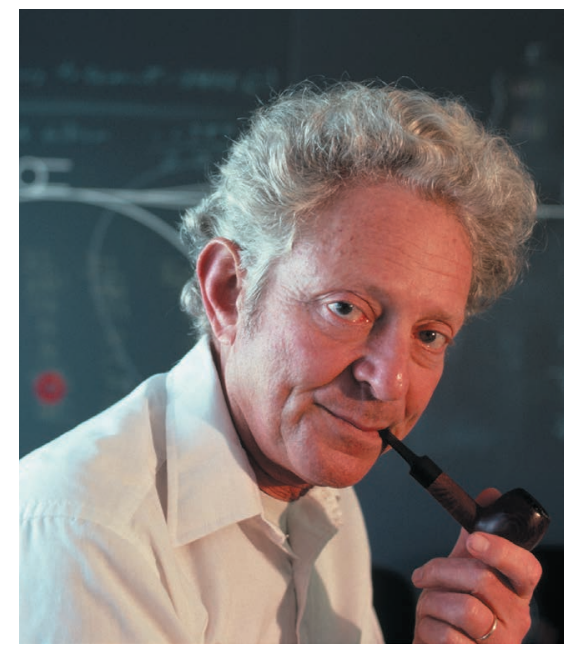

today be called a 'beam dump' experiment, the researchers aimed a powerful proton beam at a target, producing an abundance of every type of known particle. These were absorbed by a wall of dense material.

The experimenters examined the debris to see whether anything interesting emerged. It did: the muon neutrino, the second neutrino family to be discovered. The surprising discovery indicated that fundamental particles come in pairs, and advanced the idea that symmetry is intrinsic to nature's building blocks. For this work, the team shared the 1988 Nobel Prize in Physics.

Lederman conducted experiments at high-energy accelerators around the world, and contributed to the founding of the 200-gigaelectronvolt Fermi National Accelerator Laboratory (now Fermilab) in Batavia, Illinois.

Indeed, Lederman made Fermilab's first major discovery, the bottom quark, in 1977. In an elegant experiment, his team looked for particles so rare that they would result from maybe one in a hundred-trillion collisions between an intense proton beam and a target. Lederman described the new, moresensitive set-up as being as enlightening as the first telescope. They saw a signal coming from a bottom-antibottom quark pair. They named the signal upsilon, after the shape of the decay-particle trajectories, which resembled the Greek letter.

Lederman was the second director of Fermilab from 1979 to 1989 . He led the rigorous design and early operation of the Tevatron Collider (1983-2011), which was the highest-energy proton-antiproton collider in the world for nearly three decades.
From 1982, Lederman championed the most ambitious accelerator project ever the Superconducting Super Collider. But in 1993, with construction already under way, Congress cancelled the project during tightbudget years. The torch was passed to the Large Hadron Collider at CERN, Europe's particle-physics laboratory near Geneva, Switzerland. Here, the Higgs boson was discovered - its popular name in the press, the 'God Particle', was taken from the 1993 popular-science book Lederman co-wrote with Dick Teresi, The God Particle: If the Universe is the Answer, What is the Question?

Lederman leaves a lasting educational legacy. As director of Fermilab, he introduced Saturday Morning Physics, a ten-week physics class for high-school students, which is still popular more than three decades on. He started the Friends of Fermilab, a school outreach effort that grew into the Lederman Science Center at Fermilab. He founded the Illinois Mathematics and Science Academy, a residential, state-supported high school, and he championed the 'Physics First' highschool science curriculum, which teaches foundational physics before chemistry and biology.

His scientific legacy continues with ongoing efforts to explore the particles that he discovered. The neutrino, perhaps the most ubiquitous particle in the Universe, is still one of the least understood. Its tiny mass and quirky interactions remain puzzles. Alongside enormous neutrino detectors around the world, Fermilab is leading an ambitious global effort to study muon neutrinos beamed from Chicago to South Dakota, to understand how they oscillate from one flavour to another.

Several nations built dedicated accelerators to explore the bottom quark in detail. This year, Japan commissioned an ambitious new one at the KEK laboratory in Tsukuba, for example. Measurements of rare, bottomquark decays seem to be harbingers of new physics beyond the standard model.

Lederman, among others, is also credited with merging the sciences of the very small and the very big - particle physics and cosmology. Since Lederman's heyday, the study of subatomic particles has been used to probe the early Universe and its most energetic phenomena.

Nigel S. Lockyer is the director of Fermilab in Batavia, Illinois.

e-mail:lockyer@fnal.gov 